\title{
The effect of Phase I periodontal treatment on the salivary RANKL/OPG ratio in severe chronic periodontitis
}

This article was published in the following Dove Press journal:

Clinical, Cosmetic and Investigational Dentistry

Somaye Ansari Moghadam'

Somaye Sarani ${ }^{2}$

Ebrahim Alijani ${ }^{3}$

Alireza Ansari Moghadam 4

'Department of Periodontology, Oral and Dental Disease Research Center,

Zahedan University of Medical Sciences,

Zahedan, Iran; ${ }^{2}$ Oral and Dental Disease

Research Center, Zahedan University of

Medical Sciences, Zahedan, Iran;

${ }^{3}$ Department of Clinical Immunology

Research Center, Zahedan University of

Medical Sciences, Zahedan, Iran;

${ }^{4}$ Department of Health Promotion

Research Center, Zahedan University of

Medical Sciences, Zahedan, Iran
Correspondence: Somaye Sarani Department of Periodontology, Oral and

Dental Disease Research Center,

Zahedan University of Medical Sciences,

Zahedan, Iran

Tel +989333034254

Email Somaye.sarani@yahoo.com
Background: RANKL and OPG play an important role in bone resorption. The $\frac{R A N K L}{\mathrm{OPG}}$ ratio increases in periodontal disease. The present study was conducted to evaluate the effect of Phase 1 periodontal treatment on the salivary $\frac{R A N K L}{\text { OPG }}$ ratio in patients with severe chronic periodontitis.

Materials and methods: Saliva samples were collected from 13 patients with severe chronic periodontitis and 14 healthy controls at baseline and then four weeks after the treatment using unstimulated spitting. The salivary levels of RANKL, OPG and $\frac{R A N K L}{\text { OPG }}$ ratio were investigated using the ELISA method.

Results: The findings of this study showed that the mean $\frac{R A N K L}{O P G}$ ratio was significantly higher in the patients with periodontal disease than in the healthy controls $(P=0.001)$. Also, the $\frac{R A N K L}{\text { OPG }}$ ratio was significantly higher in the patients with a higher mean CAL $(P=0 / 004)$. The comparison of the salivary $\frac{R A N K L}{\text { OPG }}$ ratio in patients with periodontal disease before and four weeks after the treatment showed a significant reduction in this value four weeks after the periodontal treatment $(P=0 / 001)$.

Conclusion: The results of this study showed a direct relationship between the $\frac{R A N K L}{\text { OPG }}$ ratio and the severity of periodontal disease. Phase 1 of periodontal treatment was found to be effective in reducing the $\frac{R A N K L}{\text { OPG }}$ ratio. In other words, the $\frac{R A N K L}{\text { OPG }}$ ratio can be a good predictor of treatment success. Further long-term studies with larger sample sizes are required for confirming these results.

Keywords: chronic periodontitis, salivary levels, ratio, RANKL, OPG

\section{Introduction}

Periodontal disease is one of the most common chronic human bacterial infections that affects the supporting tissues of the teeth and is one of the main causes of tooth loss in adults. ${ }^{1}$ This disease results in the progressive degradation of the periodontal ligament (PDL) and alveolar bone together with the formation of pockets or gum recession or both. ${ }^{2,3}$ Periodontopathogens induce immunoinflammatory responses characterized by the release of inflammatory mediators. ${ }^{4}$ During the last few years new and interesting advances have been developed in periodontal treatment such as regenerative therapy, growth factors and host immuno-modulation treatment, all tools useful for tooth maintenance. ${ }^{5}$ Alveolar bone destruction is the main cause of tooth loss. The height of the alveolar bone depends on the recession and formation of the bone and the balance between these two processes and is sustained steadily. ${ }^{6}$ A new molecular system belonging to the tumor necrosis factor family has been 
identified in the regulation of bone destruction and includes three key proteins, namely the receptor activator of nuclear factor kappa-B ligand (RANKL), the receptor activator of nuclear factor kappa B (RANK) and Osteoprotegerin (OPG). OPG is a glycoprotein in the tumor necrosis factor receptor superfamily-alpha (TNFRSF). Together with RANKL and RANK, this glycoprotein forms a molecular triad that regulates bone metabolism by controlling osteoclasts. Moreover, RANKL enhances osteoclastogenesis and bone absorption by binding to RANK. OPG binds to RANKL and deactivates it and thus prevents bone absorption, and as an inhibitor, prevents RANKL interactions and thereby reduces osteoclastogenesis. ${ }^{7}$ In general, the normal metabolic activity of the bone and the stability of the bone mass depends on the balance between RANKL and OPG. ${ }^{8}$ The $\frac{R A N K L}{\text { OPG }}$ ratio determines the number of osteoclasts formed. An increase in this ratio is the main cause of bone resorption in many metabolic diseases, such as osteoporosis and periodontitis. $^{8}$ An increased $\frac{\text { RANKL }}{\text { OPG }}$ ratio is considered a sign of periodontitis and is proposed as a potential marker for the molecular diagnosis of this disease. ${ }^{9}$ The salivary $\frac{R A N K L}{\text { OPG }}$ ratio is higher in patients with a history of chronic periodontitis than in healthy people. Since RANKL and OPG changes determine the incidence of the disease and the level of response to its treatment, assessing the $\frac{R A N K L}{O P G}$ ratio is more valuable than merely evaluating OPG or RANLK by themselves. ${ }^{10}$

Saliva is a very good sampling source that is easily collected and contains local and cystic markers as well as different enzymes and enzymatic and immunological products. These biomarkers are very extensive and different articles have emphasized their role in the periodontal disease. It thus appears that the regulation of these biomarkers can offer an acceptable solution for periodontal challenges. ${ }^{11}$ For example The treatment of infrabony defects with hyaluronic acid offered an additional benefit in terms of CAL gain, PD reduction, and predictability compared to treatment with open flap debridement. $^{12}$

Given the contradictory results of studies on the $\frac{R A N K L}{\text { OPG }}$ ratio in periodontal patients and the changes occurring in the $\frac{R A N K L}{\text { OPG }}$ ratio after periodontal treatment, the present study was conducted to determine the effect of Phase 1 of periodontal treatment on the $\frac{R A N K L}{\text { OPG }}$ ratio in periodontal patients. We did this study with the hypothesis that periodontal treatment did not change the $\frac{R A N K L}{\text { OPG }}$ ratio.

\section{Materials and methods}

This study was conducted in accordance with the Declaration of Helsinki. A permission was first obtained from the Ethics Committee of Zahedan University of Medical Sciences and the longitudinal study was registered and the project begun (IR.ZAUMS.REC.1396.291). In this study, a periodontal chart was filled out for 13 individuals presenting to the Department of Periodontology in Zahedan Dental School after their examination and upon the specialist's approval. The candidates were recruited for the study if they received a diagnosis of severe chronic periodontitis and after they were briefed on the project and submitted their written consent. Healthy controls $(n=14)$ were selected from the patients' relatives. A medical history was also taken from this group and they entered the study after submitting their written consent and undergoing a clinical examination. Participants' data, including age, gender and BMI, were recorded in information forms.

\section{Case group}

The patients were diagnosed with periodontitis based on their probing depth measurements using a Williams probe, clinical attachment level and clinical signs of inflammation. The patients with a CAL $\geq 5 \mathrm{~mm}$ in more than $30 \%$ of their oral sites, bone resorption in the radiographs of at least five teeth in at least two quadrants and gingivitis symptoms such as bleeding during probing and gum color change were taken as patients with severe chronic periodontitis. $^{13}$

\section{Control group}

The periodontally-healthy people with a probing depth less than $3 \mathrm{~mm}$ in all their teeth, PI $\leq 10 \%$ and no clinical symptoms of gingivitis were taken as the controls. ${ }^{14}$

The case and control groups were homogenized in terms of age, gender and BMI (Tables 1 and 2). The

Table I The mean age and BMI of the healthy controls and patients

\begin{tabular}{|l|l|l|l|}
\hline Variable & Group & Mean \pm SD & $\begin{array}{l}\text { Significance } \\
\text { level }\end{array}$ \\
\hline Mean age & $\begin{array}{l}\text { Healthy controls } \\
\text { Patient }\end{array}$ & $\begin{array}{l}33 / 64 \pm 7 / 08 \\
37 / 46 \pm 9 / 8\end{array}$ & $0 / 96$ \\
\hline Mean BMI & Healthy controls & $20 / 14 \pm 9 / 8$ & $0 / 1$ \\
& Patient & $20 / 00 \pm 0.91$ & \\
\hline
\end{tabular}


Table 2 The frequency distribution of gender in the two groups

\begin{tabular}{|l|l|l|l|l|l|}
\hline \multirow{2}{*}{ Group } & \multicolumn{2}{|l|}{} & \multirow{2}{*}{ P-value for the Chi-Square Test } \\
\hline & \multicolumn{2}{|l|}{ Gender } & \multicolumn{2}{|l}{ Male } & \\
\cline { 2 - 5 } & Female & Number & Percentage & Number & $0 / 84$ \\
Patients & Percentage & 7 & $46 / 2$ & 6 & \\
Healthy controls & $53 / 8$ & 7 & 50 & 7 & \\
\hline
\end{tabular}

inclusion criteria for the case group consisted of having severe chronic periodontitis with $\mathrm{CAL} \geq 5 \mathrm{~mm}$, being at least 20 years old, having more than 20 remaining teeth, being systemically healthy, not having consumed any medications such as antibiotics or anti-inflammatory medications over the last three months and not having received any periodontal treatments over the last year. ${ }^{13}$ The exclusion criteria for the case group consisted of Unwillingness of the patient to continue cooperation; Failure to follow the guidelines of the investigators; Perform other treatments,use of drugs affecting the results of the study during the study pregnancy, having less than 22 permanent teeth and smoking. ${ }^{13,23}$

The inclusion criteria for the control group consisted of not having gingivitis, a plaque index of $10 \%$ or and a PD equal to or less than $3 \mathrm{~mm} .{ }^{15}$ Saliva samples were collected from the two groups at baseline and four weeks after the SRP treatment for OPG and RANKL measurements. The patients then underwent Phase 1 of periodontal treatment. All the patients underwent scaling, root planing and brushing. Handheld ultrasonic instruments and rubber cup and prophylaxis paste were used for this purpose.

\section{Saliva collection}

The best method for collecting saliva is the unstimulated method, since in the stimulated method, elements may be released into the saliva that could change its qualitative composition. ${ }^{6}$

Unstimulated saliva samples were collected by spitting. The participants were asked to avoid eating, drinking and brushing their teeth 90 mins prior to the sampling. All the samples were collected from 9 to $11 \mathrm{am}$. During the sample collection, the participants were in a seated position and slightly leaned forward and emptied their saliva in a pre-sterilized test tube (gamma counter) one to two times per minute over a ten-minute period. ${ }^{16}$ Three milliliters of saliva were collected from each participant. ${ }^{6}$ This method is called unstimulated saliva collection and is a reliable method for testing the saliva content. ${ }^{17}$
The collected salivary samples were stored at $-70{ }^{\circ} \mathrm{C}$ until the ELISA test was performed.

OPG and RANKL measurement using the ELISA method:

The Human OPG ELISA Kit (Cat. No. CK-E90916) and Human RANKL ELISA Kit (Cat. No. CK-E30391) made by EASTBIOPHARM (USA) were used to measure the salivary OPG and RANK levels.

In the laboratory analysis stage, the saliva samples were first centrifuged for four minutes at $6000 \mathrm{rpm}$ after reaching room temperature to sediment the salivary compounds that could confound the test results. The samples and standards were distributed in microplates pre-coated with specific OPG and RANKL monoclonal antibodies in the corresponding kits. The procedures were performed according to the kit instructions. After washing to remove unbound material, an enzyme-linked polyclonal antibody specific for RANKL and OPG was added to the wells. After a second wash with buffer, chromogen solution A $50 \mu \mathrm{L}$, then chromogen solution B $50 \mu \mathrm{L}$ was added to each well and incubated for $10 \mathrm{~min}$ at $37{ }^{\circ} \mathrm{C}$ away from light. The reactions were stopped with the appropriate substrate reaction (the blue changes into yellow immediately). The optical density of the color in each well was measured at $450 \mathrm{~nm}$. RANKL and OPG levels were reported as mean $\pm \mathrm{SD}$, expressed in $\mathrm{pg} / \mathrm{mL}$. The $\mathrm{OPG} /$ RANKL ratio was also calculated. ${ }^{18}$

\section{Results}

The aim of this study was to determine the effect of Phase 1 of periodontal treatment on the $\frac{R A N K L}{\text { OPG }}$ ratio in patients with periodontal disease. The mean age was 33.64 \pm 7.08 years in the healthy controls and $37.46 \pm 9.8$ years in the patients. The mean BMI was $20.14 \pm 9.8$ in the healthy controls and $20.00 \pm 0.91$ in the patients (Table 1).

According to the results of Table 1, the mean age and BMI distribution was normal in the case and control groups as per the Kolmogorov-Smirnov test results. Given the normality of the results, the independent $t$-test 
was used for the analysis. This test showed no significant differences between the two groups in terms of the mean age and BMI.

Table 2 presents the gender distribution in the two groups. According to the data, $53.8 \%$ of the patients and $50 \%$ of the healthy controls were female, and $46.2 \%$ of the patients and $50 \%$ of the healthy controls were male. The Chi-square test showed no statistically significant differences between the two groups $(P=0 / 84)$.

The comparison of the mean $\frac{R A N K L}{\mathrm{OPG}}$ ratio between the patients and the healthy controls using the $t$-test showed a significantly higher value in the patients with periodontal disease compared to the healthy controls $(P=0 / 001)$ (Table 3).

In order to evaluate the effect of the mean CAL on the salivary $\frac{R A N K L}{\mathrm{OPG}}$ ratio in the patients with periodontal disease, they were divided into two groups in terms of their mean $\mathrm{CAL}$, including a $\mathrm{CAL}<5$ group and a $\mathrm{CAL} \geq 5$ group. The two groups were then compared in terms of their $\frac{R A N K L}{\text { OPG }}$ ratio. Table 4 presents these findings. The $t$-test showed a significant difference between the mean CAL of the patients with periodontal disease in terms of $\frac{R A N K L}{\mathrm{OPG}}$. In other words, the $\frac{R A N K L}{\text { OPG }}$ showed a significant increase as the mean CAL increased in the patients $(P=0 / 004)$ (Table 4$)$.

Based on Table 5, the $\frac{R A N K L}{\text { OPG }}$ ratio decreased significantly four weeks after the non-surgical periodontal treatment compared to baseline $(P=0 / 001)$.

In addition, the $\frac{R A N K L}{\mathrm{OPG}}$ ratio was still significantly higher in the patients than in the healthy controls despite its significant reduction in the case group four weeks after the treatment $(P<0 / 0001)$ (Table 6$)$.

Table 3 The salivary $\frac{R A N K L}{O P G}$ ratio in the patients and healthy controls

\begin{tabular}{|c|c|c|}
\hline Group & $\frac{R A N K L}{\mathrm{OPG}}$ mean \pm SD & $P$-value \\
\hline $\begin{array}{l}\text { Healthy controls } \\
\text { Patients before the treatment }\end{array}$ & $\begin{array}{l}|/ 86 \pm| / 5 \mid \\
625 / 46 \pm 495 / 42\end{array}$ & $0 / 001$ \\
\hline
\end{tabular}

Table 4 A comparison of the mean CAL with the salivary $\frac{\text { RANKL }}{\text { OPG }}$ ratio in the patients with periodontal disease before the periodontal treatment

\begin{tabular}{|l|l|l|}
\hline Group & $\frac{\mathrm{RANKL}}{\mathrm{OPG}}$ mean \pm SD & P-value \\
\hline $\mathrm{CAL} \geq 5$ & $1149 / 38 \pm 345 / 64$ & $0 / 004$ \\
$\mathrm{CAL}<5$ & $392 / 83 \pm 353 / 75$ & \\
\hline
\end{tabular}

Table 5 The salivary $\frac{R A N K L}{\mathrm{OPG}}$ ratio in the patients with periodontal disease four weeks after the treatment

\begin{tabular}{|c|c|c|}
\hline Group Mean $\frac{R A N K L}{\mathrm{OPG}}$ & $\begin{array}{l}\frac{R A N K L}{\mathrm{OPG}} \\
\text { mean } \pm \mathbf{S D}\end{array}$ & $\begin{array}{l}P \text { - } \\
\text { value }\end{array}$ \\
\hline $\begin{array}{l}\text { Patients with periodontal disease } \\
\text { Patients with periodontal disease four } \\
\text { weeks after the treatment }\end{array}$ & $\begin{array}{l}625 / 61 \pm 495 / 35 \\
57 / 54 \pm 39 / 78\end{array}$ & $0 / 001$ \\
\hline
\end{tabular}

Table 6 The salivary $\frac{R A N K L}{\text { OPG }}$ ratio of the healthy controls and patients with periodontal disease four weeks after the treatment

\begin{tabular}{|l|l|l|}
\hline Group & $\begin{array}{l}\frac{R A N K L}{\mathrm{OPG}} \\
\text { mean } \pm \text { SD }\end{array}$ & $\begin{array}{l}\boldsymbol{P} \text { - } \\
\text { value }\end{array}$ \\
\hline $\begin{array}{l}\text { Healthy controls } \\
\text { Patients with periodontal disease four } \\
\text { weeks after the treatment }\end{array}$ & $\begin{array}{l}1 / 8 \pm 61 / 5 \mathrm{I} \\
57 / 54 \pm 39 / 78\end{array}$ & $\begin{array}{l}P<0 / \\
000 \mathrm{I}\end{array}$ \\
\hline
\end{tabular}

\section{Discussion}

The $\frac{R A N K L}{\text { OPG }}$ ratio has a potential diagnostic value for periodontitis and can be a good predictor of treatment success.

In the present study, the mean $\frac{R A N K L}{\text { OPG }}$ ratio was significantly higher in the patients with severe chronic periodontitis than in the healthy controls $(P=0 / 001)$.

This finding is in line with the results of studies by Costa et $\mathrm{al},{ }^{19}$ Mogi et $\mathrm{al}^{23}$ Tobon-Arroyave et $\mathrm{al},{ }^{21}$ Baltacioğlu et $\mathrm{al}^{22}{ }^{22}$ Tabari et $\mathrm{al}^{20}$ and Ochanji et al. ${ }^{24}$

In studies by Costa et al, ${ }^{19}$ Tabari et $\mathrm{al}^{20}$ and TobonArroyave et al, ${ }^{21}$ the $\frac{R A N K L}{\text { OPG }}$ ratio was significantly higher in the patients with chronic periodontitis compared to the healthy controls $(P<0.001, P<0.05$ and $P<0.05)$. The present findings are consistent with the results of these three studies.

The present findings are also consistent with the results obtained by Baltacioğlu et al. ${ }^{22}$ In this study, the $\frac{R A N K L}{\text { OPG }}$ ratio was significantly higher in the patients with chronic and generalized aggressive periodontitis than in the healthy controls $(P<0.05)$.

Moreover, Mogi et $\mathrm{al}^{23}$ and Ochanji et $\mathrm{al}^{24}$ found that the $\frac{R A N K L}{\mathrm{OPG}}$ ratio is higher in periodontitis patients with any degree of illness compared to healthy people $(P<0.01$ and $P<0.001)$. The present findings concur with the results obtained by these researchers.

Ghasemi et $\mathrm{al}^{25}$ however, found no statistically significant differences between the salivary $\frac{R A N K L}{\mathrm{OPG}}$ ratio among the four diabetic groups (with and without periodontitis) and the healthy participants (with and without periodontitis) examined $(P<0.05)$. This result contradicts 
the present findings. This inconsistency could be due to the differences in sample size, as Ghasemi's study was conducted on 50 volunteers, including 27 type-1 diabetes patients, 12 of whom had periodontitis, and 23 systemically-healthy participants, 12 of whom had periodontitis. The present study, however, was conducted on 13 patients with chronic periodontitis and 14 healthy controls. This inconsistency could also be due to the differences in sampling method, sensitivity and specificity of the ELISA kit and the various levels of clinical inflammation.

Sarlati et $\mathrm{al}^{26}$ found that the GCF $\frac{R A N K L}{\mathrm{OPG}}$ ratio was not significantly different between the three groups examined, ie the healthy, chronic periodontitis and invasive periodontitis groups $(P=0.41)$. This result is not in line with the present findings. This inconsistency could be due to the fact that Sarlati et al studied the RANKL and OPG concentration in Gingival Crevicular Fluid (GCF), while the present study investigated the salivary concentration of RANKL and OPG. The GCF around the entire teeth in the mouth and the secreted cytokines are present in a whole saliva sample, and the periodontium condition is not necessarily the same around all the teeth. Meanwhile, GCF is collected in a site-specific and selective manner from sites with periodontitis in the mouth. Moreover, the sampling might not have been performed from the actual site of the disease. In addition, in GCF, the RANKL is found in very small amounts, and even then, only in a form bonded with OPG or RANK, and this observation might explain the lack of statistically significant differences in this study.

In the present study, the $\frac{R A N K L}{\text { OPG }}$ ratio was significantly higher in the patients with severe chronic periodontitis with a higher mean CAL compared to those with a lower CAL $(P=0 / 004)$.

In 2016, Ochanji et $\mathrm{al}^{24}$ found a strong correlation between the $\frac{R A N K L}{\text { OPG }}$ ratio and the severity of chronic periodontitis $(P<0.001)$. Their findings are consistent with the results of the present study.

There was also a direct correlation between CAL and RANKL and the $\frac{R A N K L}{\text { OPG }}$ ratio according to Bostanci et al, ${ }^{27}$ Tabari et $\mathrm{al}^{20}$ and Ghasemi et $\mathrm{al}^{25}(P<0.01, P=0.015$ and $P<0.05)$. The results of these three studies were thus consistent with the present findings, but contrary to the results reported by Bostanci et al. ${ }^{10}$ According to their study, there was a negative correlation between $\mathrm{CAL}$ and the $\frac{R A N K L}{\mathrm{OPG}}$ ratio in chronic periodontitis, ${ }^{10}$ although this relationship was not statistically significant. The reason for this inconsistency may be the differences in the $\frac{R A N K L}{\mathrm{OPG}}$ ratio measurement, since they examined the $\frac{R A N K L}{\mathrm{OPG}}$ ratio in the GCF.

Comparing the salivary $\frac{R A N K L}{\mathrm{OPG}}$ ratio in patients with severe chronic periodontitis before and four weeks after the treatment showed a significant reduction in this value after four weeks of treatment $(P=0 / 001)$.

Based on the results obtained by Santos et $\mathrm{al}^{28}$ three and six months after treatment, the $\frac{R A N K L}{\mathrm{OPG}}$ ratio reduced in the patients with controlled diabetes and periodontitis, as contrary to the patients with uncontrolled diabetes and periodontitis $(P<0.05)$, as this ratio had increased in those with uncontrolled diabetes. The reason for this disparity might be the effect of diabetes on the $\frac{R A N K L}{\text { OPG }}$ ratio, since people with diabetes have less bone repair potential during the active or degenerative stages of periodontitis. Nonetheless, since the $\frac{R A N K L}{O P G}$ ratio had reduced in the controlled diabetes group after the treatment, these results are considered consistent with the present findings.

Moreover, according to a study by Bostanci et al, ${ }^{10}$ despite the improvement in clinical symptoms, the GCF $\frac{R A N K L}{\mathrm{OPG}}$ ratio remained unchanged two, three and four months after non-surgical periodontal treatment $(P<0.01)$.

In a study by Buduneli et al, OPG decreased four weeks after the periodontal treatment, but no significant changes were observed in the RANKL levels, and an increase was thus observed in the GCF $\frac{R A N K L}{\text { OPG }}$ ratio. $^{29}$

The results of these two studies are in contradiction with the present study. This disparity, may be due to the fact that samples were taken from the GCF in these two studies but in the present study samples were taken from saliva, also in the study by Bostanci et $\mathrm{al}^{10}$ samples were taken two, three and four months after treatment but the present study samples were taken 1 month after periodontal treatment and restoration pattern vary depending on the time elapsed from the treatment.

In the present study, the mean $\frac{R A N K L}{\mathrm{OPG}}$ ratio was significantly higher in the patients with periodontal disease four weeks after the treatment compared to the healthy controls $(P<0 / 0001)$. This finding is consistent with the results reported by Dereka et al, ${ }^{30}$ in which the $\frac{R A N K L}{\text { OPG }}$ ratio was higher in the patients with severe chronic periodontitis who had received non-surgical periodontal treatment four to six weeks prior to sampling compared to the healthy participants, although this difference was not statistically significant. The fact that this difference was statistically significant in the present study suggests that although 
periodontal treatment changes the $\frac{R A N K L}{\text { OPG }}$ ratio, this value is still higher in these patients compared to healthy people. Future studies are therefore recommended to use longer follow-up periods.

\section{Conclusion}

The findings of this study show that the salivary $\frac{R A N K L}{\text { OPG }}$ ratio is increased in patients with severe chronic periodontitis in comparison with healthy people, and Phase 1 of periodontal treatment is effective in decreasing the $\frac{R A N K L}{\mathrm{OPG}}$ ratio. In other words, the $\frac{R A N K L}{\mathrm{OPG}}$ ratio has a potential diagnostic value for periodontitis and can be a good predictor of treatment success. Further and more expansive studies are required in order to confirm this hypothesis.

\section{Disclosure}

The authors report no conflicts of interest in this work.

\section{References}

1. Su F, Liu -S-S, Ma J-L, Wang D-S, Ling-Ling E, Liu H-C. Enhancement of periodontal tissue regeneration by transplantation of osteoprotegerin-engineered periodontal ligament stem cells. Stem Cell Res Ther. 2015;6(1):22. doi:10.1186/s13287-015-0023-3

2. Shum I, Leung P-C, Kwok A, et al. Periodontal conditions in elderly men with and without osteoporosis or osteopenia. J Periodontol. 2010;81(10):1396-1402. doi:10.1902/jop.2010.100052

3. Payne W, Page RC, Ogilvie A, Hall W. Histopathologic features of the initial and early stages of experimental gingivitis in man. $J$ Periodontal Res. 1975;10(2):51-64.

4. Nokhbehsaim M, Memmert S, Damanaki A, et al. Effect of interleukin-1 $\beta$ on ghrelin receptor in periodontal cells. Clin Oral Investig. 2019;23(1):113-122. doi:10.1007/s00784-018-2417-y

5. Matarese G, Ramaglia L, Fiorillo L, Cervino G, Lauritano F, Isola G. Implantology and periodontal disease: the panacea to problem solving? Open Dent J. 2017;30(11):460-465. doi:10.2174/1874210601711010460

6. Oliver RC, Brown LJ, Löe H. Periodontal diseases in the United States population. J Periodontol. 1998;69(2):269-278. doi:10.1902/ jop.1998.69.2.269

7. Farzanegi P, Ebrahimi K, Habibian M. The effect of resistance training circuit with Medicago sativa extracts on levels of osteoprotegerin and nuclear factor of Kappa-B in thin girls. Pars Jahrom Univ Med Sci. 2016;14(3):27-34. doi:10.29252/jmj.14.3.27

8. Pfaffe T, Cooper-White J, Beyerlein P, Kostner K, Punyadeera C. Diagnostic potential of saliva: current state and future applications. Clin Chem. 2011;57(5):675-687. doi:10.1373/clinchem.2010.153767

9. Belibasakis GN, Bostanci N. The RANKL-OPG system in clinical periodontology. $J$ Clin Periodontol. 2012;39(3):239-248. doi:10.1111/j.1600-051X.2011.01810.x

10. Bostanci N, Saygan B, Emingil G, Atilla G, Belibasakis GN. Effect of periodontal treatment on receptor activator of NF- $\mathrm{KB}$ ligand and osteoprotegerin levels and relative ratio in gingival crevicular fluid. $J$ Clin Periodontol. 2011;38(5):428-433. doi:10.1111/j.1600051X.2011.01701.x
11. Jahangirneghad M, Yousefimanesh H, Vesali M. Evaluation of osteoprotegrin concentration in the saliva of patients with generalized moderate chronic periodontitis. J Mashhad Dent Sch. 2012;36 (4):279-284.

12. Briguglio F, Briguglio E, Briguglio R, Cafiero C, Isola G. Treatment of infrabony periodontal defects using a resorbable biopolymer of hyaluronic acid: a randomized clinical trial. Quintessence Int. 2013;44(3):231-240. doi:10.3290/j.qi.a29054

13. Hassan SH, El-Refai MI, Ghallab NA, Kasem RF, Shaker OG. Effect of periodontal surgery on osteoprotegerin levels in gingival crevicular fluid, saliva, and gingival tissues of chronic periodontitis patients. Dis Markers. 2015;2015:341259. doi:10.1155/2015/341259

14. Miller CS, King CP, Langub MC, Kryscio RJ, Thomas MV. Salivary biomarkers of existing periodontal disease: a cross-sectional study. $J$ Am Dent Assoc. 2006;137(3):322-329.

15. Sadeghi R, Nazari Moghaddam K, Jooyandeh J. Clinical evaluation of endodotic therapy on periodontal tissue healing in chronic advanced periodontitis. J Dent Med. 2004;17(3):83-91.

16. Agha-Hosseini F, Mirzaii-Dizgah I, Moghaddam P, Akrad Z. Stimulated whole salivary flow rate and composition in menopausal women with oral dryness feeling. Oral Dis. 2007;13(3):320-323. doi:10.1111/j.1601-0825.2006.01288.x

17. Bretz WA, Loesche WJ, Chen YM, Schork MA, Dominguez BL, Grossman N. Minor salivary gland secretion in the elderly. Oral Surg Oral Med Oral Pathol Oral Radiol Endodontics. 2000;89(6):696701. doi:10.1067/moe.2000.105329

18. Kapasa ER, Giannoudis PV, Jia X, Hatton PV, Yang XB. The effect of RANKL/OPG balance on reducing implant complications. $J$ Funct Biomater. 2017;8(4). pii: E42. doi:10.3390/jfb8040042.

19. Costa LC, Fonseca M, Pinheiro A, et al. Chronic periodontitis and RANKL/OPG ratio in peri-implant mucosae inflammation. Braz Dent J. 2018;29(1):14-22. doi:10.1590/0103-6440201801241

20. Tabari AZ, Azadmehr A, Tabrizi MAA, Hamissi J, Ghaedi FB. Salivary soluble receptor activator of nuclear factor kappa B ligand/osteoprotegerin ratio in periodontal disease and health. $J$ Periodontal Implant Sci. 2013;43(5):227-232. doi:10.5051/ jpis.2013.43.5.227

21. Tobón-Arroyave SI, Isaza-Guzmán DM, Restrepo-Cadavid EM, Zapata-Molina SM, Martínez-Pabón MC. Association of salivary levels of the bone remodelling regulators sRANKL and OPG with periodontal clinical status. J Clin Periodontol. 2012;39(12):11321140. doi:10.1111/jcpe. 12012

22. Baltacioğlu E, Kehribar MA, Yuva P, et al. Total oxidant status and bone resorption biomarkers in serum and gingival crevicular fluid of patients with periodontitis. J Periodontol. 2014;85(2):317-326. doi:10.1902/jop.2013.130012

23. Mogi M, Otogoto J, Ota N, Togari A. Differential expression of RANKL and osteoprotegerin in gingival crevicular fluid of patients with periodontitis. J Dent Res. 2004;83(2):166-169. doi:10.1177/ 154405910408300216

24. Ochanji A, Matu N, Mulli T. Association of salivary RANKL and osteoprotegerin levels with periodontal health. Clin Exp Dent Res. 2017;3(2):45-50. doi:10.1002/cre2.49

25. Ghasemi M, Sattari M, Khamseh ME, Kavoli S. Relationship of salivary RANKL and OPG with periodontal disease in type 1 diabetic patients. $J$ Islamic Dent Assoc IRAN (JIDA). 2011;23(2):95-103.

26. Sarlati F, Sattari M, Razzaghi S, Nasiri M. Receptor activator of nuclear factor kappa B ligand and osteoprotegerin levels in gingival crevicular fluid. Dent Res J (Isfahan). 2012;9(6):752.

27. Bostanci N, Ilgenli T, Emingil G, et al. Differential expression of receptor activator of nuclear factor-kappaB ligand and osteoprotegerin mRNA in periodontal diseases. J Periodontal Res. 2007;42 (4):287-293. doi:10.1111/j.1600-0765.2006.00946.x 
28. Santos VR, Lima JA, Gonçalves TED, et al. Receptor activator of nuclear factor-Kappa B Ligand/osteoprotegerin ratio in sites of chronic periodontitis of subjects with poorly and well-controlled type 2 diabetes. J Periodontol. 2010;81(10):1455-1465. doi:10.1902/ jop.2010.100125

29. Buduneli N, Buduneli E, Kütükçüler N. Interleukin-17, RANKL, and osteoprotegerin levels in gingival crevicular fluid from smoking and non-smoking patients with chronic periodontitis during initial periodontal treatment. J Periodontol. 2009;80(8):1274-1280. doi:10.1902/ jop.2009.090106
30. Dereka XE, Markopoulou CE, Fanourakis G, Tseleni-Balafouta S, Vrotsos IA. RANKL and OPG mRNA level after non-surgical periodontal treatment. Inflammation. 2010;33(3):200-206. doi:10.1007/ s10753-009-9174-7

Clinical, Cosmetic and Investigational Dentistry

\section{Publish your work in this journal}

Clinical, Cosmetic and Investigational Dentistry is an international, peer-reviewed, open access, online journal focusing on the latest clinical and experimental research in dentistry with specific emphasis on cosmetic interventions. Innovative developments in dental materials, techniques and devices that improve outcomes and patient satisfaction and preference will be highlighted. The manuscript management system is completely online and includes a very quick and fair peer-review system, which is all easy to use. Visit http://www.dovepress.com/testimonials.php to read real quotes from published authors. 